\section{MS}

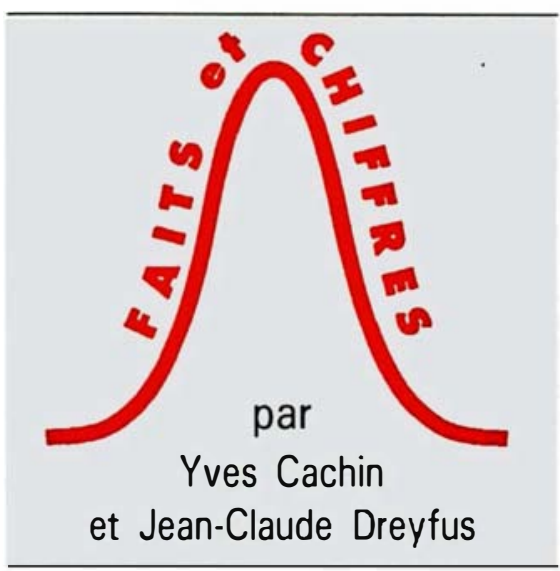

\section{Remerciements}

Nous remercions Mme le Docteur Hélène Sancho-Garnier, directeur de l'U.135 de l'Inserm, chef du département de biostatistique et d'épidémiologie, à l'obligeance de qui nous devons ces documents.

\title{
Statistiques de mortalité en France pour 1990. Les cancers comparés aux autres causes aux autres causes
}

\begin{abstract}
(2)
\end{abstract}




\begin{tabular}{|c|c|c|c|c|c|c|c|c|c|c|c|c|c|}
\hline \multicolumn{14}{|c|}{ NOMBRE DE DÉCĖS PAR SEXE ET PAR TRANCHE D'ÂGE } \\
\hline \multirow{2}{*}{$\begin{array}{c}\text { France } 1990 \\
\begin{array}{c}\text { Causes médicales de décès } \\
\text { No de la Classification internationale } \\
\text { des maladies, ge révision }\end{array}\end{array}$} & \multicolumn{10}{|c|}{ Résultats définitifs } & \multicolumn{3}{|c|}{ Liste simplifiée S9 - Inserm } \\
\hline & Sexe & $\begin{array}{c}\text { Total } \\
\text { tous àges }\end{array}$ & $\begin{array}{l}\text { Moins } \\
\text { d'un an }\end{array}$ & $\begin{array}{c}1 \text { à } 4 \\
\text { ans }\end{array}$ & $\begin{array}{c}5 \text { à } 14 \\
\text { ans }\end{array}$ & $\begin{array}{l}15 \text { à } 24 \\
\text { ans }\end{array}$ & $\begin{array}{c}25 \text { à } 34 \\
\text { ans }\end{array}$ & $\begin{array}{c}35 \text { à } 44 \\
\text { ans }\end{array}$ & $\begin{array}{c}45 \text { à } 54 \\
\text { ans }\end{array}$ & $\begin{array}{c}55 \text { à } 64 \\
\text { ans }\end{array}$ & $\begin{array}{c}65 \text { à } 74 \\
\text { ans }\end{array}$ & $\begin{array}{c}75 \text { à } 84 \\
\text { ans }\end{array}$ & $\begin{array}{l}85 \text { ans } \\
\text { et plus }\end{array}$ \\
\hline $\begin{array}{l}149 \text { Tumeurs malignes des lèvres, de la } \\
\text { cavité buccale et du pharynx }\end{array}$ & $\begin{array}{l}M \\
F\end{array}$ & $\begin{array}{c}4865 \\
672\end{array}$ & - & - & $\begin{array}{l}1 \\
-\end{array}$ & $\begin{array}{l}6 \\
3\end{array}$ & $\begin{array}{l}21 \\
6\end{array}$ & $\begin{array}{c}332 \\
41\end{array}$ & $\begin{array}{c}943 \\
81\end{array}$ & $\begin{array}{c}1690 \\
150\end{array}$ & $\begin{array}{c}1085 \\
130\end{array}$ & $\begin{array}{l}644 \\
134\end{array}$ & $\begin{array}{l}143 \\
127\end{array}$ \\
\hline Tumeur maligne de l'œsophage & $\begin{array}{c}M \\
F\end{array}$ & $\begin{array}{c}4311 \\
705\end{array}$ & - & - & - & - & $\begin{array}{l}3 \\
1\end{array}$ & $\begin{array}{c}147 \\
16\end{array}$ & $\begin{array}{c}580 \\
52\end{array}$ & $\begin{array}{c}1456 \\
121\end{array}$ & $\begin{array}{c}1164 \\
134\end{array}$ & $\begin{array}{l}752 \\
242\end{array}$ & $\begin{array}{l}209 \\
139\end{array}$ \\
\hline Tumeur maligne de l'estomac & $\begin{array}{l}M \\
F\end{array}$ & $\begin{array}{l}3962 \\
2836\end{array}$ & $\begin{array}{l}- \\
-\end{array}$ & - & $\begin{array}{l}- \\
-\end{array}$ & $\overline{1}$ & $\begin{array}{c}6 \\
15\end{array}$ & $\begin{array}{l}91 \\
46\end{array}$ & $\begin{array}{l}226 \\
73\end{array}$ & $\begin{array}{l}684 \\
231\end{array}$ & $\begin{array}{c}1021 \\
442\end{array}$ & $\begin{array}{l}1385 \\
1103\end{array}$ & $\begin{array}{l}549 \\
925\end{array}$ \\
\hline Tumeur maligne de l'intestin & $\begin{array}{c}M \\
F\end{array}$ & $\begin{array}{l}8035 \\
7725\end{array}$ & 1 & - & - & $\begin{array}{l}5 \\
2\end{array}$ & $\begin{array}{l}20 \\
27\end{array}$ & $\begin{array}{l}142 \\
106\end{array}$ & $\begin{array}{l}367 \\
271\end{array}$ & $\begin{array}{c}1361 \\
870\end{array}$ & $\begin{array}{l}2154 \\
1468\end{array}$ & $\begin{array}{l}2837 \\
2794\end{array}$ & $\begin{array}{ll}1 & 148 \\
2 & 187\end{array}$ \\
\hline Tumeur maligne du pancréas & $\begin{array}{c}M \\
F\end{array}$ & $\begin{array}{l}3090 \\
2647\end{array}$ & - & - & $\overline{1}$ & $\overline{2}$ & $\begin{array}{c}11 \\
7\end{array}$ & $\begin{array}{l}81 \\
35\end{array}$ & $\begin{array}{c}230 \\
90\end{array}$ & $\begin{array}{l}690 \\
320\end{array}$ & $\begin{array}{l}910 \\
567\end{array}$ & $\begin{array}{c}901 \\
1019\end{array}$ & $\begin{array}{l}267 \\
606\end{array}$ \\
\hline $\begin{array}{l}\text { Tumeurs malignes d'autres parties, } \\
\text { appareil digestif et péritoine }\end{array}$ & $\begin{array}{c}M \\
F\end{array}$ & $\begin{array}{l}6539 \\
4229\end{array}$ & $\begin{array}{l}2 \\
-\end{array}$ & $\begin{array}{l}4 \\
4\end{array}$ & $\begin{array}{l}2 \\
2\end{array}$ & $\begin{array}{l}8 \\
3\end{array}$ & $\begin{array}{l}23 \\
10\end{array}$ & $\begin{array}{c}102 \\
49\end{array}$ & $\begin{array}{l}390 \\
137\end{array}$ & $\begin{array}{c}1540 \\
487\end{array}$ & $\begin{array}{c}2092 \\
843\end{array}$ & $\begin{array}{l}1806 \\
1513\end{array}$ & $\begin{array}{c}570 \\
1181\end{array}$ \\
\hline Tumeur maligne du larynx & $\begin{array}{c}M \\
F\end{array}$ & $\begin{array}{c}2880 \\
155\end{array}$ & - & - & - & - & 2 & $\begin{array}{c}107 \\
5\end{array}$ & $\begin{array}{c}438 \\
27\end{array}$ & $\begin{array}{c}954 \\
39\end{array}$ & $\begin{array}{c}783 \\
28\end{array}$ & $\begin{array}{c}484 \\
40\end{array}$ & $\begin{array}{l}112 \\
16\end{array}$ \\
\hline $\begin{array}{l}\text { Tumeur maligne de la trachée, des } \\
\text { bronches et du poumon }\end{array}$ & $\begin{array}{c}M \\
F\end{array}$ & $\begin{array}{l}18805 \\
2812\end{array}$ & $\begin{array}{l}2 \\
-\end{array}$ & $\begin{array}{l}2 \\
-\end{array}$ & $\begin{array}{l}1 \\
-\end{array}$ & $\begin{array}{l}2 \\
2\end{array}$ & $\begin{array}{l}51 \\
17\end{array}$ & $\begin{array}{l}645 \\
103\end{array}$ & $\begin{array}{c}1932 \\
218\end{array}$ & $\begin{array}{c}5149 \\
590\end{array}$ & $\begin{array}{c}5665 \\
719\end{array}$ & $\begin{array}{c}4378 \\
815\end{array}$ & $\begin{array}{l}978 \\
348\end{array}$ \\
\hline $\begin{array}{l}\text { Tumeurs malignes d'autres parties, } \\
\text { app. resp. et org. thoraciques }\end{array}$ & $\begin{array}{c}M \\
F\end{array}$ & $\begin{array}{c}3211 \\
586\end{array}$ & - & - & 2 & $\begin{array}{l}3 \\
3\end{array}$ & $\begin{array}{c}23 \\
7\end{array}$ & $\begin{array}{l}168 \\
27\end{array}$ & $\begin{array}{c}521 \\
55\end{array}$ & $\begin{array}{c}1100 \\
110\end{array}$ & $\begin{array}{l}771 \\
126\end{array}$ & $\begin{array}{l}516 \\
181\end{array}$ & $\begin{array}{l}107 \\
77\end{array}$ \\
\hline $\begin{array}{l}\text { Tumeur maligne des os et du carti- } \\
\text { lage articulaire }\end{array}$ & $\begin{array}{c}M \\
F\end{array}$ & $\begin{array}{l}448 \\
275\end{array}$ & - & $\begin{array}{l}4 \\
1\end{array}$ & $\begin{array}{l}6 \\
6\end{array}$ & $\begin{array}{l}29 \\
18\end{array}$ & $\begin{array}{l}13 \\
14\end{array}$ & $\begin{array}{l}18 \\
18\end{array}$ & $\begin{array}{l}49 \\
10\end{array}$ & $\begin{array}{c}110 \\
29\end{array}$ & $\begin{array}{l}96 \\
51\end{array}$ & $\begin{array}{l}90 \\
77\end{array}$ & $\begin{array}{l}33 \\
51\end{array}$ \\
\hline $\begin{array}{l}\text { Tumeur maligne du tissu conjonctif } \\
\text { et des autres tissus mous }\end{array}$ & $\begin{array}{c}M \\
F\end{array}$ & $\begin{array}{l}292 \\
265\end{array}$ & 1 & $\begin{array}{l}4 \\
2\end{array}$ & $\begin{array}{c}8 \\
10\end{array}$ & $\begin{array}{l}14 \\
15\end{array}$ & $\begin{array}{l}18 \\
15\end{array}$ & $\begin{array}{l}22 \\
24\end{array}$ & $\begin{array}{l}33 \\
19\end{array}$ & $\begin{array}{l}55 \\
33\end{array}$ & $\begin{array}{l}52 \\
44\end{array}$ & $\begin{array}{l}60 \\
58\end{array}$ & $\begin{array}{l}25 \\
45\end{array}$ \\
\hline Tumeur maligne de la peau & $\begin{array}{c}M \\
F\end{array}$ & $\begin{array}{l}710 \\
689\end{array}$ & - & - & - & $\begin{array}{l}4 \\
5\end{array}$ & $\begin{array}{l}23 \\
27\end{array}$ & $\begin{array}{l}60 \\
55\end{array}$ & $\begin{array}{l}70 \\
75\end{array}$ & $\begin{array}{c}140 \\
92\end{array}$ & $\begin{array}{l}156 \\
105\end{array}$ & $\begin{array}{l}160 \\
166\end{array}$ & $\begin{array}{c}97 \\
164\end{array}$ \\
\hline Tumeur maligne du sein & $\begin{array}{c}M \\
F\end{array}$ & $\begin{array}{c}129 \\
10173\end{array}$ & - & - & - & $\overline{2}$ & $\begin{array}{c}2 \\
77\end{array}$ & $\begin{array}{c}6 \\
636\end{array}$ & $\begin{array}{c}14 \\
1205\end{array}$ & $\begin{array}{c}26 \\
2168\end{array}$ & $\begin{array}{c}29 \\
2191\end{array}$ & $\begin{array}{c}37 \\
2437\end{array}$ & $\begin{array}{c}15 \\
1457\end{array}$ \\
\hline 180-182 Tumeur maligne de l'utérus & $\begin{array}{l}M \\
F\end{array}$ & $3 \overline{139}$ & - & - & - & $\overline{2}$ & $\overline{32}$ & - & $\frac{-}{235}$ & - & $\overline{731}$ & - & $-\overline{488}$ \\
\hline Tumeur maligne de la prostate & $\begin{array}{l}M \\
F\end{array}$ & $\begin{array}{r}9211 \\
-\end{array}$ & - & - & - & $\begin{array}{c}2 \\
-\end{array}$ & $\begin{array}{l}3 \\
-\end{array}$ & $\begin{array}{l}7 \\
-\end{array}$ & $\begin{array}{c}62 \\
-\end{array}$ & $\begin{array}{r}631 \\
-\end{array}$ & $\begin{array}{r}1881 \\
-\end{array}$ & $\begin{array}{r}4300 \\
-\end{array}$ & $\begin{array}{r}2325 \\
-\end{array}$ \\
\hline 188 Tumeur maligne de la vessie & $\begin{array}{l}M \\
F\end{array}$ & $\begin{array}{l}3170 \\
1105\end{array}$ & - & - & - & $\overline{1}$ & $\begin{array}{l}1 \\
3\end{array}$ & $\begin{array}{r}21 \\
4\end{array}$ & $\begin{array}{r}145 \\
19\end{array}$ & $\begin{array}{r}546 \\
64\end{array}$ & $\begin{array}{l}805 \\
166\end{array}$ & $\begin{array}{r}1144 \\
475\end{array}$ & $\begin{array}{l}508 \\
373\end{array}$ \\
\hline $\begin{array}{l}189 \text { Tumeur maligne du rein et d'organes } \\
\text { urinaires autres ou n.p. }\end{array}$ & $\begin{array}{c}M \\
F\end{array}$ & $\begin{array}{l}1987 \\
1142\end{array}$ & $\begin{array}{l}2 \\
1\end{array}$ & $\begin{array}{l}2 \\
3\end{array}$ & $\begin{array}{l}4 \\
2\end{array}$ & $\begin{array}{l}5 \\
1\end{array}$ & $\begin{array}{r}10 \\
6\end{array}$ & $\begin{array}{l}45 \\
22\end{array}$ & $\begin{array}{r}140 \\
65\end{array}$ & $\begin{array}{l}435 \\
165\end{array}$ & $\begin{array}{l}555 \\
255\end{array}$ & $\begin{array}{l}599 \\
405\end{array}$ & $\begin{array}{l}190 \\
217\end{array}$ \\
\hline $\begin{array}{l}\text { 181, } 184 \text { Tumeurs malignes d'autres organes, } \\
\text { génito-urinaire } \\
186,187 \text { app. }\end{array}$ & $\begin{array}{c}M \\
F\end{array}$ & $\begin{array}{l}214 \\
528\end{array}$ & - & $\begin{array}{l}1 \\
2\end{array}$ & $\begin{array}{r}1 \\
-\end{array}$ & $\begin{array}{r}18 \\
2\end{array}$ & $\begin{array}{r}37 \\
1\end{array}$ & $\begin{array}{l}32 \\
10\end{array}$ & $\begin{array}{l}13 \\
11\end{array}$ & $\begin{array}{l}25 \\
63\end{array}$ & $\begin{array}{l}25 \\
95\end{array}$ & $\begin{array}{r}43 \\
186\end{array}$ & $\begin{array}{r}19 \\
158\end{array}$ \\
\hline 191 Tumeur maligne de l'encéphale & $\begin{array}{c}M \\
F\end{array}$ & $\begin{array}{r}1367 \\
973\end{array}$ & $\begin{array}{l}1 \\
1\end{array}$ & $\begin{array}{r}10 \\
9\end{array}$ & $\begin{array}{l}42 \\
24\end{array}$ & $\begin{array}{l}27 \\
18\end{array}$ & $\begin{array}{l}52 \\
39\end{array}$ & $\begin{array}{r}133 \\
70\end{array}$ & $\begin{array}{r}179 \\
92\end{array}$ & $\begin{array}{l}353 \\
226\end{array}$ & $\begin{array}{l}332 \\
263\end{array}$ & $\begin{array}{l}204 \\
178\end{array}$ & $\begin{array}{l}34 \\
53\end{array}$ \\
\hline $\begin{array}{l}\text { 190, 192-199 Tumeurs malignes de sièges, } \\
\text { autres et sans précision }\end{array}$ & $\begin{array}{l}M \\
F\end{array}$ & $\begin{array}{l}5746 \\
5206\end{array}$ & $\begin{array}{l}5 \\
3\end{array}$ & $\begin{array}{l}12 \\
12\end{array}$ & $\begin{array}{r}13 \\
8\end{array}$ & $\begin{array}{l}16 \\
15\end{array}$ & $\begin{array}{l}35 \\
24\end{array}$ & $\begin{array}{l}186 \\
125\end{array}$ & $\begin{array}{l}472 \\
249\end{array}$ & $\begin{array}{r}1292 \\
632\end{array}$ & $\begin{array}{l}1465 \\
1026\end{array}$ & $\begin{array}{l}1630 \\
1085\end{array}$ & $\begin{array}{r}620 \\
1307\end{array}$ \\
\hline Maladie de Hodgkin & $\begin{array}{c}M \\
F\end{array}$ & $\begin{array}{l}228 \\
109\end{array}$ & - & - & $\begin{array}{c}1 \\
-\end{array}$ & $\begin{array}{l}8 \\
6\end{array}$ & $\begin{array}{l}20 \\
11\end{array}$ & $\begin{array}{l}43 \\
15\end{array}$ & $\begin{array}{r}35 \\
7\end{array}$ & $\begin{array}{r}31 \\
9\end{array}$ & $\begin{array}{l}35 \\
15\end{array}$ & $\begin{array}{l}46 \\
34\end{array}$ & $\begin{array}{r}9 \\
12\end{array}$ \\
\hline
\end{tabular}




\section{NOMBRE DE DÉCĖS PAR SEXE ET PAR TRANCHE D'ÂGE}

\section{France 1990}

Causes médicales de décès

$N^{0}$ de la Classification internationale des maladies, ge révision
Résultats définitifs

Liste simplifiè 59 - Inserm

\begin{tabular}{|c|c|c|c|c|c|c|c|c|c|c|c|c|c|}
\hline 200, 202 Autres tumeurs malignes des tissus & M & 1657 & - & 4 & 7 & 22 & 46 & 87 & 138 & 300 & 439 & 475 & 139 \\
\hline
\end{tabular}

lymphoïde et histiocytaire

Myélome multiple et tumeurs
immunoprolifératives

204-208Leucémies

\begin{tabular}{|c|c|c|c|c|} 
Sexe & $\begin{array}{c}\text { Total } \\
\text { tous âges }\end{array}$ & $\begin{array}{c}\text { Moins } \\
\text { d'un an }\end{array}$ & $\begin{array}{c}1 \text { à } 4 \\
\text { ans }\end{array}$ & $\begin{array}{c}5 \text { à } 14 \\
\text { ans }\end{array}$ \\
\hline M & 1657 & - & 4 & 7 \\
F & 1458 & 1 & 1 & 8
\end{tabular}

15 à

M 830

$\begin{array}{ll}M & 830 \\ F & 956\end{array}$

\begin{tabular}{|c|c|c|c|c|c|c|c|c|c|c|c|c|c|}
\hline 2u4-zuoleucem & $\mathrm{M}$ & $\begin{array}{l}2515 \\
2232\end{array}$ & $\begin{array}{l}5 \\
4\end{array}$ & $\begin{array}{l}31 \\
19\end{array}$ & $\begin{array}{l}54 \\
43\end{array}$ & $\begin{array}{l}16 \\
45\end{array}$ & $\begin{array}{l}88 \\
44\end{array}$ & $\begin{array}{r}115 \\
89\end{array}$ & 118 & $\begin{array}{l}349 \\
238\end{array}$ & $\begin{array}{l}553 \\
385\end{array}$ & 721 & $\begin{array}{l}344 \\
526\end{array}$ \\
\hline 210-229Tumeurs bénignes & $\begin{array}{c}M \\
F\end{array}$ & $\begin{array}{l}210 \\
299\end{array}$ & $\begin{array}{l}1 \\
2\end{array}$ & $\begin{array}{c}4 \\
-\end{array}$ & $\begin{array}{l}4 \\
1\end{array}$ & $\begin{array}{l}4 \\
1\end{array}$ & $\begin{array}{l}7 \\
6\end{array}$ & $\begin{array}{r}8 \\
17\end{array}$ & $\begin{array}{l}12 \\
21\end{array}$ & $\begin{array}{l}28 \\
29\end{array}$ & $\begin{array}{l}46 \\
54\end{array}$ & $\begin{array}{r}80 \\
105\end{array}$ & $\begin{array}{l}16 \\
63\end{array}$ \\
\hline $\begin{array}{l}\text { 230-239Carcinome in situ, tumeurs à évolu- } \\
\text { tion imprévisible et de nature n.p. }\end{array}$ & $\begin{array}{l}M \\
F\end{array}$ & $\begin{array}{l}1754 \\
1620\end{array}$ & $\begin{array}{l}5 \\
3\end{array}$ & $\begin{array}{l}12 \\
11\end{array}$ & $\begin{array}{l}22 \\
25\end{array}$ & $\begin{array}{l}16 \\
13\end{array}$ & $\begin{array}{l}35 \\
34\end{array}$ & $\begin{array}{l}79 \\
52\end{array}$ & $\begin{array}{r}112 \\
69\end{array}$ & $\begin{array}{l}275 \\
167\end{array}$ & $\begin{array}{l}402 \\
253\end{array}$ & $\begin{array}{l}542 \\
525\end{array}$ & $\begin{array}{l}254 \\
468\end{array}$ \\
\hline $\begin{array}{l}\text { 240-279Maladies endocriniennes, nutrition, } \\
\text { métabolisme, troubles immunitaires }\end{array}$ & $\begin{array}{c}M \\
F\end{array}$ & $\begin{array}{l}5042 \\
8291\end{array}$ & $\begin{array}{l}41 \\
32\end{array}$ & $\begin{array}{l}21 \\
12\end{array}$ & $\begin{array}{l}22 \\
30\end{array}$ & $\begin{array}{l}28 \\
17\end{array}$ & $\begin{array}{l}49 \\
27\end{array}$ & $\begin{array}{r}116 \\
44\end{array}$ & $\begin{array}{r}193 \\
98\end{array}$ & $\begin{array}{l}509 \\
350\end{array}$ & $\begin{array}{l}868 \\
873\end{array}$ & $\begin{array}{l}1752 \\
2784\end{array}$ & $\begin{array}{l}1443 \\
4024\end{array}$ \\
\hline $\begin{array}{l}\text { 280-289Maladies du sang et des organes } \\
\text { hématopoïétiques }\end{array}$ & $\begin{array}{c}M \\
F\end{array}$ & $\begin{array}{l}1193 \\
1377\end{array}$ & $\begin{array}{r}11 \\
8\end{array}$ & $\begin{array}{r}8 \\
14\end{array}$ & $\begin{array}{l}9 \\
9\end{array}$ & $\begin{array}{r}12 \\
5\end{array}$ & $\begin{array}{l}13 \\
12\end{array}$ & $\begin{array}{l}27 \\
19\end{array}$ & $\begin{array}{l}42 \\
21\end{array}$ & $\begin{array}{r}117 \\
68\end{array}$ & $\begin{array}{l}218 \\
139\end{array}$ & $\begin{array}{l}438 \\
447\end{array}$ & $\begin{array}{l}298 \\
635\end{array}$ \\
\hline
\end{tabular}

\begin{tabular}{|l|l|}
\hline$M$ & 2515 \\
$F$ & 2232
\end{tabular}

\begin{tabular}{c|c|c|}
\hline- & - & - \\
- & - & 1
\end{tabular}

\begin{tabular}{l|l|}
12 & 27 \\
\hline
\end{tabular}

46
12
7

\begin{tabular}{l|l|}
59 & 127 \\
25 & 116
\end{tabular}

201

291 139

\begin{tabular}{|c|c|c|c|c|c|c|c|c|c|c|c|c|c|}
\hline $\begin{array}{l}\text { 280-289Maladies du sang et des organes } \\
\text { hématopoiétiques }\end{array}$ & $\begin{array}{l}M \\
F\end{array}$ & $\begin{array}{l}1193 \\
1377\end{array}$ & $\begin{array}{r}11 \\
8\end{array}$ & $\begin{array}{r}8 \\
14\end{array}$ & $\begin{array}{l}9 \\
9\end{array}$ & $\begin{array}{r}12 \\
5\end{array}$ & $\begin{array}{l}13 \\
12\end{array}$ & $\begin{array}{l}27 \\
19\end{array}$ & $\begin{array}{l}42 \\
21\end{array}$ & $\begin{array}{r}117 \\
68\end{array}$ & $\begin{array}{l}218 \\
139\end{array}$ & $\begin{array}{l}438 \\
447\end{array}$ & $\begin{array}{l}298 \\
635\end{array}$ \\
\hline 290-319Troubles mentaux & $\begin{array}{c}M \\
F\end{array}$ & $\begin{array}{l}5485 \\
7427\end{array}$ & - & $\begin{array}{l}1 \\
1\end{array}$ & - & $\begin{array}{l}78 \\
22\end{array}$ & $\begin{array}{r}231 \\
57\end{array}$ & $\begin{array}{l}433 \\
126\end{array}$ & $\begin{array}{l}481 \\
145\end{array}$ & $\begin{array}{l}834 \\
250\end{array}$ & $\begin{array}{l}696 \\
391\end{array}$ & $\begin{array}{l}1492 \\
2343\end{array}$ & $\begin{array}{l}1239 \\
4092\end{array}$ \\
\hline 320-389Maladies du système nerveux et des & M & 5419 & 81 & 55 & 63 & 142 & 140 & 249 & 287 & 561 & 1050 & 1951 & 840 \\
\hline
\end{tabular}
320-389Maladies du système nerveux et des
organes des sens

390-459Maladies de l'appareil circulatoire

\begin{tabular}{|l|r|r|r|r|r|r|r|r|r|r|r|r|}
\hline $\mathrm{M}$ & 5419 & 81 & 55 & 63 & 142 & 140 & 249 & 287 & 561 & 1050 & 1951 & 840 \\
$\mathrm{~F}$ & 5874 & 60 & 36 & 40 & 62 & 74 & 116 & 147 & 467 & 895 & 2209 & 1768 \\
\hline
\end{tabular}

$249 \quad 287$

\begin{tabular}{|l|r|r|r|r|r|r|r|r|r|r|r|r|}
\hline $\mathrm{M}$ & 5419 & 81 & 55 & 63 & 142 & 140 & 249 & 287 & 561 & 1050 & 1951 & 840 \\
$\mathrm{~F}$ & 5874 & 60 & 36 & 40 & 62 & 74 & 116 & 147 & 467 & 895 & 2209 & 1768 \\
\hline
\end{tabular}

460-519Maladies de l'appareil respiratoire

\begin{tabular}{l|l} 
M & 79357
\end{tabular}

\begin{tabular}{|l|l|l|}
49 & 20 & 30 \\
39 & 18 & 18
\end{tabular}

\begin{tabular}{r|r|r}
133 & 416 & 1735
\end{tabular}

\begin{tabular}{l|l|l|l|l|l|}
735 & 3066 & 8883 & 15534 & 30249
\end{tabular}

\begin{tabular}{l|r}
$F$ & 95187 \\
\hline
\end{tabular}

F 18286

\begin{tabular}{|c|c|c|c|c|c|c|c|}
\hline 520-579Maladies de l'appareil digestif & $\begin{array}{c}M \\
F\end{array}$ & $\begin{array}{l}14486 \\
12596\end{array}$ & $\begin{array}{l}37 \\
19\end{array}$ & $\begin{array}{l}8 \\
9\end{array}$ & $\begin{array}{r}6 \\
12\end{array}$ & $\begin{array}{l}26 \\
11\end{array}$ & $\begin{array}{r}173 \\
73\end{array}$ \\
\hline $\begin{array}{c}\text { 580-629Maladies des organes } \\
\text { génito-urinaires }\end{array}$ & $\begin{array}{c}M \\
F\end{array}$ & $\begin{array}{l}3615 \\
3563\end{array}$ & $\begin{array}{l}4 \\
5\end{array}$ & $\begin{array}{r}4 \\
-\end{array}$ & $\begin{array}{l}1 \\
2\end{array}$ & $\begin{array}{l}6 \\
3\end{array}$ & $\begin{array}{l}14 \\
11\end{array}$ \\
\hline
\end{tabular}

\begin{tabular}{|c|c|}
\hline 630-676Complications grossesse, accouche- \\
ment, suites de couches
\end{tabular}

\begin{tabular}{|c|c|}
\hline $\begin{array}{c}\text { 680-709Maladies de la peau et du tissu cel- } \\
\text { lulaire sous-cutané }\end{array}$ \\
\hline
\end{tabular}

M

F 79

\begin{tabular}{l|r}
$M$ & 638 \\
$F$ & 1462
\end{tabular}

710-739Mal. du système ostéo-articulaire,
muscles, tissu conjonctif

740-759Anomalies congénitales

\begin{tabular}{|r|r}
\hline$M$ & 8 \\
$F$ & 1735
\end{tabular}

\begin{tabular}{|c|c|c|c|c|c|c|c|c|c|c|c|c|c|}
\hline 740-759Anomalies congénitales & $\begin{array}{l}M \\
F\end{array}$ & $\begin{array}{l}975 \\
844\end{array}$ & $\begin{array}{l}620 \\
512\end{array}$ & $\begin{array}{l}80 \\
71\end{array}$ & $\begin{array}{l}38 \\
42\end{array}$ & $\begin{array}{l}48 \\
31\end{array}$ & $\begin{array}{l}39 \\
32\end{array}$ & $\begin{array}{l}35 \\
29\end{array}$ & $\begin{array}{l}32 \\
18\end{array}$ & $\begin{array}{l}32 \\
33\end{array}$ & $\begin{array}{l}24 \\
31\end{array}$ & $\begin{array}{l}20 \\
32\end{array}$ & $\begin{array}{r}7 \\
13\end{array}$ \\
\hline $\begin{array}{l}\text { 760-779Affections dont I'origine se situe } \\
\text { dans la période périnatale }\end{array}$ & $\begin{array}{l}M \\
F\end{array}$ & $\begin{array}{l}829 \\
598\end{array}$ & $\begin{array}{l}823 \\
595\end{array}$ & $\begin{array}{r}4 \\
-\end{array}$ & $\begin{array}{l}1 \\
3\end{array}$ & $\begin{array}{c}1 \\
-\end{array}$ & - & - & - & - & - & - & - \\
\hline $\begin{array}{l}\text { 780-799Symptômes, signes et états morbi- } \\
\text { des mal définis }\end{array}$ & $\begin{array}{l}M \\
F\end{array}$ & $\begin{array}{l}14020 \\
17448\end{array}$ & $\begin{array}{r}1285 \\
818\end{array}$ & $\begin{array}{l}67 \\
31\end{array}$ & $\begin{array}{l}38 \\
22\end{array}$ & $\begin{array}{l}357 \\
130\end{array}$ & $\begin{array}{l}749 \\
234\end{array}$ & $\begin{array}{l}878 \\
297\end{array}$ & $\begin{array}{l}890 \\
290\end{array}$ & $\begin{array}{r}1374 \\
482\end{array}$ & $\begin{array}{r}1649 \\
931\end{array}$ & $\begin{array}{l}3173 \\
4045\end{array}$ & $\begin{array}{r}3560 \\
10168\end{array}$ \\
\hline $\begin{array}{l}\text { E800-E999 Causes extérieures de trauma- } \\
\text { tismes et empoisonnements }\end{array}$ & $\begin{array}{l}M \\
F\end{array}$ & $\begin{array}{l}29039 \\
19215\end{array}$ & $\begin{array}{l}189 \\
127\end{array}$ & $\begin{array}{l}279 \\
160\end{array}$ & $\begin{array}{l}372 \\
224\end{array}$ & $\begin{array}{r}3715 \\
944\end{array}$ & $\begin{array}{l}4087 \\
1059\end{array}$ & $\begin{array}{l}3993 \\
1194\end{array}$ & $\begin{array}{l}3160 \\
1136\end{array}$ & $\begin{array}{l}3357 \\
1442\end{array}$ & $\begin{array}{l}3038 \\
1700\end{array}$ & $\begin{array}{l}4036 \\
4400\end{array}$ & $\begin{array}{l}2813 \\
6829\end{array}$ \\
\hline Total toutes causes & $\begin{array}{l}M \\
F\end{array}$ & $\begin{array}{l}272664 \\
253537\end{array}$ & $\begin{array}{l}3284 \\
2315\end{array}$ & $\begin{array}{l}696 \\
463\end{array}$ & $\begin{array}{l}789 \\
568\end{array}$ & $\begin{array}{l}4964 \\
1581\end{array}$ & $\begin{array}{l}7451 \\
2589\end{array}$ & $\begin{array}{r}12339 \\
4988\end{array}$ & $\begin{array}{r}17976 \\
7373\end{array}$ & $\begin{array}{l}40705 \\
16626\end{array}$ & $\begin{array}{l}53562 \\
28564\end{array}$ & $\begin{array}{l}81609 \\
81932\end{array}$ & $\begin{array}{r}49289 \\
106538\end{array}$ \\
\hline
\end{tabular}

